BMJ Surgery, Interventions, \& Health Technologies

\section{Transesophageal echocardiography and risk of respiratory failure in patients who had ischemic stroke or transient ischemic attack: an IDEAL phase 4 study}

To cite: Bruce SS, Navi BB, Zhang C, et al. Transesophageal echocardiography and risk of respiratory failure in patients who had ischemic stroke or transient ischemic attack: an IDEAL phase 4 study. BMJ Surg Interv Health Technologies 2022;4:e000116. doi:10.1136/ bmjsit-2021-000116

Received 16 September 2021 Accepted 24 January 2022

Check for updates

(c) Author(s) (or their employer(s)) 2022. Re-use permitted under CC BY-NC. No commercial re-use. See rights and permissions. Published by BMJ.

${ }^{1}$ Clinical and Translational Neuroscience Unit, Department of Neurology and Feil Family Brain and Mind Research Institute, Weill Cornell Medicine, New York, New York, USA ${ }^{2}$ Division of Cardiology, Weill Cornell Medicine, New York, New York, USA

${ }^{3}$ Division of Pulmonary and Critical Care Medicine, Weill Cornell Medicine, New York, New York, USA

${ }^{4}$ Department of Population Health Sciences, Weill Cornell Medicine, New York, New York, USA

Correspondence to

Dr Hooman Kamel;

hok9010@med.cornell.edu

\section{ABSTRACT}

Objective Transesophageal echocardiography (TEE) is sometimes used to search for cardioembolic sources after ischemic stroke or transient ischemic attack (TIA). TEE visualizes some sources better than transthoracic echocardiography, but TEE is invasive and may cause aspiration. Few data exist on the risk of respiratory complications after TEE in patients who had stroke or TIA. Our objective was to determine whether TEE was associated with increased risk of respiratory failure in patients who had ischemic stroke or TIA.

Design This is a retrospective cohort study using administrative data from inpatient and outpatient insurance claims collected by the US federal government's Centers for Medicare and Medicaid Services.

Setting Hospitals and outpatient clinics throughout the USA.

Participants 99081 patients $\geq 65$ years old hospitalized for out-of-hospital ischemic stroke or TIA, defined by validated International Classification of Disease- $9 / 10$ diagnosis codes and present-on-admission codes, using claims data from 2008 to 2018 in a random 5\% sample of Medicare beneficiaries. Main outcome measures Acute respiratory failure, defined as endotracheal intubation and/or mechanical ventilation, starting on the first day after admission through 28 days afterward.

Results of 99081 patients included in this analysis, $73733(74.4 \%)$ had an ischemic stroke and 25348 (25.6\%) a TIA. TEE was performed in 4677 (4.7\%) patients and intubation and/or mechanical ventilation in 1403 (1.4\%) patients. The 28 -day cumulative risk of respiratory failure after TEE $(1.4 \% ; 95 \% \mathrm{Cl} 0.8 \%$ to $2.7 \%)$ was similar to that seen in those without TEE $(1.4 \% ; 95 \% \mathrm{Cl} 1.4 \%$ to $1.5 \%)(p=0.84)$. After adjustment for age, sex, race, Charlson comorbidities, diagnosis of stroke versus TIA, intravenous thrombolysis, and mechanical thrombectomy, TEE was not associated with an increased risk of respiratory failure ( $\mathrm{HR}, 0.9 ; 95 \% \mathrm{Cl} 0.6$ to 1.2 ). Conclusions In a cohort of older patients who had ischemic stroke or TIA, TEE was not associated with an increased risk of subsequent respiratory failure.

\section{INTRODUCTION}

Ischemic stroke is a major cause of death and disability. In patients hospitalized for
Key messages

What is already known about this subject?

- Transesophageal echocardiography (TEE) is frequently performed in patients who had stroke or transient ischemic attack to look for sources of cardioembolism.

- Although case series suggest that TEE is generally safe, few data exist specifically regarding respiratory complications associated with the procedure.

What are the new findings?

- In a large sample of Medicare beneficiaries who had ischemic stroke or transient ischemic attack, we found that TEE was not associated with an increased risk of respiratory failure.

\section{How might these results affect future} research or surgical practice?

- These results may help clinicians weigh the potential risks and benefits of TEE for etiologic evaluation after ischemic stroke or transient ischemic attack.

ischemic stroke, respiratory complications are among the most common causes of morbidity and mortality. ${ }^{1}$ Patients who had stroke have a relatively high risk of respiratory failure given frequent frailty, comorbid conditions, reduced awareness, and impaired motor control of swallowing and cough.

Transesophageal echocardiography (TEE) is sometimes performed as part of the evaluation of ischemic stroke or transient ischemic attack (TIA) to assess for potential sources of cardiac embolism. ${ }^{3}$ Unlike transthoracic echocardiography, which is performed more frequently, ${ }^{3}$ TEE is an invasive procedure. Although the procedure can be performed without sedation, various levels of sedation are often administered, ranging from conscious sedation to general anesthesia. ${ }^{4}$ Case series suggest that TEE is generally safe after ischemic stroke and TIA. ${ }^{5-9}$ However, there are few safety data involving control patients who 
had stroke or TIA who did not undergo TEE, especially regarding severe but rare complications such as aspiration leading to respiratory failure. Therefore, we used a large sample of Medicare claims to determine whether TEE is associated with an increased risk of respiratory failure in patients who had ischemic stroke or TIA.

\section{METHODS}

\section{Design}

We performed a retrospective cohort study using inpatient and outpatient claims from 2008 through 2018 from a 5\% random sample of Medicare beneficiaries. The US federal government's Centers for Medicare and Medicaid Services (CMS) provides health insurance to most US residents once they reach 65 years of age. CMS makes available research-specific limited data sets on claims submitted by providers and hospitals in the course of Medicare beneficiaries' clinical care. ${ }^{10}$ Such data on outpatient physician visits are available for only a $5 \%$ random sample of Medicare beneficiaries. Claims data from hospitals include dates of hospitalization and International Classification of Diseases (ICD) diagnosis and procedure codes. Physician claims include Current Procedural Terminology (CPT) codes, the dates of service, and physicians' specialty. Multiple claims for a given patient can be linked via a unique beneficiary identifier code, thus allowing for a comprehensive and longitudinal analysis of each beneficiary's care over time. The data used for this analysis cannot be directly shared by the authors under the terms of their data use agreement, but the data can be obtained by application to CMS. This study was not preregistered.

\section{Patient population}

We included patients $\geq 65$ years of age with continuous coverage in traditional fee-for-service Medicare (both parts A and B) for at least 1 year (or until death, if applicable) between 2008 through 2018. From this sample, we selected patients hospitalized between 2009 and 2018 for acute ischemic stroke and TIA. Between January 1, 2009 and September 30, 2015, the diagnosis of stroke was defined by an ICD-9 diagnosis code algorithm previously validated to have a sensitivity of $86 \%$, specificity of $95 \%$, and positive predictive value of $90 \%$ when compared with medical record review, ${ }^{11}$ and TIA was defined by ICD-9 code 435.x, which has been previously validated to have a positive predictive value of $70 \%$ or greater. ${ }^{12}$ After September 30, 2015, we used ICD-10 code I.63 for ischemic stroke and ICD-10 code G45 for TIA; both codes have also been validated as highly reliable. ${ }^{13}$ Since we were interested in the use of TEE for evaluation of routine, community-onset strokes, we limited our population to hospitalizations with a primary discharge diagnosis of stroke that was documented as present on admission. Present-on-admission codes in hospital discharge data have been previously validated. ${ }^{14}$ The start date of January 1, 2009 was chosen because 2008 was used as a run-in period to ascertain comorbidities and to exclude patients without at least 1 year of Medicare coverage prior to their first stroke. For patients who had multiple ischemic stroke hospitalizations during the study period, we examined only the first documented stroke hospitalization (index hospitalization).

\section{Measurements}

We used ICD and CPT procedure codes to determine relevant procedures. TEE was defined as CPT codes 93312, 93313, or 93314. Respiratory failure was defined as CPT code 31500 for endotracheal intubation or ICD-9 procedure codes $96.70-96.72$ or ICD-10 procedure codes 5A093-5A095 or 5A193-5A195 for mechanical ventilation. As covariates, we identified intravenous thrombolysis, defined using ICD-9 procedure code 99.10 or ICD-10 procedure code 3E03017 or 3E03317; mechanical thrombectomy, defined using CPT code 37184 or ICD-9 procedure code 39.74 or ICD-10 procedure code 03CG3, 03CH3, 03CI3, 03CJ3, 03CK3, 03CL3, 03CP3, or 03CQ3; and Charlson comorbidities, defined using previously validated ICD-9 and ICD-10 codes. ${ }^{15}$ For descriptive purposes, we ascertained the following vascular risk factors and comorbidities: atrial fibrillation, hypertension, diabetes mellitus, coronary heart disease, heart failure, peripheral vascular disease, chronic kidney disease, valvular heart disease, chronic obstructive pulmonary disease, tobacco use, alcohol abuse, infective endocarditis, and cardioversion. The National Institutes of Health Stroke Scale (NIHSS) score was ascertained for the subset of patients with NIHSS scores documented in their ICD-10 hospital discharge codes. ${ }^{16}$

\section{Statistical analysis}

Descriptive statistics are reported as mean $\pm \mathrm{SD}$ or percentages with $95 \%$ CI. We performed Kaplan-Meier survival analysis, with patients entering the risk period for respiratory failure on the day after the index hospital admission and exiting the risk period 28 days afterward. We included episodes of respiratory failure during hospitalizations subsequent to the index hospitalization as long as the outcome occurred within 28 days of the index hospital admission. Patients were censored at the time of death. The procedural codes identifying TEE, intubation, and mechanical ventilation had exact dates associated with them; however, if these procedures were performed on the same day, there is no way to determine which procedure came first. Thus, we conservatively did not count TEE performed on the same day as, or after, endotracheal intubation and/or initiation of mechanical ventilation to avoid a spurious association from TEEs being performed in already intubated patients. We included TEEs starting on the day of the index hospital admission up through the last day of the index hospitalization, with the goal of addressing whether TEE on day $\mathrm{X}$ of the stroke hospitalization was associated with respiratory failure on day $\mathrm{X}+1$ onwards through day 28. TEE was modeled as a time-varying variable, so patients who 
underwent TEE contributed time-at-risk to the control group before and on the day of their TEE and then to the TEE group starting on the day after their TEE. Cumulative rates of respiratory failure after TEE versus those without TEE were compared using the log-rank test. Cox proportional hazards analysis was performed to determine the association between TEE and the risk of acute respiratory failure after adjustment for age, race, sex, number of Charlson comorbidities, diagnosis (stroke vs TIA), intravenous thrombolysis, and mechanical thrombectomy. We performed a subgroup analysis stratified by index diagnosis (stroke vs TIA). Statistical analyses were performed using Stata V.15.

\section{Sensitivity analyses}

We used our fully adjusted model to perform five sensitivity analyses. First, we excluded patients who underwent cardioversion or had a diagnosis of infective endocarditis. Second, we included as our outcome only cases of intubation and/or mechanical ventilation that resulted in tracheostomy to limit our outcome to the most severe and consequential cases of respiratory failure. Third, we excluded patients who died during the index hospitalization or were discharged to hospice. Fourth, we added a TEE-calendar year interaction term to the model to determine whether the relationship between TEE and respiratory failure changed over time. Finally, we included patients who experienced respiratory failure on the same day as TEE.

\section{RESULTS}

Of 99081 patients included in this analysis, 73733 (74.4\%) had an ischemic stroke and $25348(25.6 \%)$ a TIA. TEE was performed in $4677(4.7 \%)$ patients, 4137 of whom had an ischemic stroke and 540 had a TIA. Patients who underwent TEE were younger, were more often male, less often had atrial fibrillation and congestive heart failure, more often had valvular heart disease and infective endocarditis, and had higher rates of treatment with thrombolysis, thrombectomy, and cardioversion; in the subset of 5434 patients with available NIHSS data, the NIHSS scores of patients who did versus did not undergo TEE were similar (table 1). During the study period, 1403 (1.4\%) underwent intubation and/or mechanical ventilation starting on the day after admission and through 28 days afterward. Those with respiratory failure were younger but had more vascular risk factors and comorbidities than those who did not require intubation or mechanical ventilation; in the subset of patients with available NIHSS data, the NIHSS scores of patients who had respiratory failure were substantially higher than those without respiratory failure (table 2).

The cumulative risk of respiratory failure at 28 days after TEE $(1.4 \%$; $95 \%$ CI $0.8 \%$ to $2.7 \%$ ) was similar to those who did not undergo TEE (1.4\%; 95\% CI $1.4 \%$ to $1.5 \%)$ ( $\mathrm{p}=0.84$ ) (figure 1). In an unadjusted model, TEE was not associated with risk of intubation and/or mechanical
Table 1 Characteristics of Medicare beneficiaries who had ischemic stroke or transient ischemic attack, stratified by whether TEE was performed ( $5 \%$ national sample)

\begin{tabular}{|c|c|c|}
\hline Characteristics* $^{*}$ & TEE $(n=4677)$ & $\begin{array}{l}\text { No TEE } \\
(n=94404)\end{array}$ \\
\hline Age, years, mean (SD) & $75.3(6.9)$ & $79.4(8.3)$ \\
\hline Female & $2338(50.0)$ & $55437(58.7)$ \\
\hline \multicolumn{3}{|l|}{ Race } \\
\hline White & $4000(85.5)$ & $79873(84.6)$ \\
\hline Black & $480(10.3)$ & $9813(10.4)$ \\
\hline Other & $197(4.2)$ & $4718(5.0)$ \\
\hline $\begin{array}{l}\text { Charlson comorbidities, } \\
\text { number, mean (SD) }\end{array}$ & $2.8(1.6)$ & $2.9(1.6)$ \\
\hline $\begin{array}{l}\text { NIHSS score, median } \\
(\mathrm{IQR}) \dagger\end{array}$ & $3(1-7)$ & $4(1-8)$ \\
\hline Intravenous thrombolysis & $299(6.4)$ & $4212(4.5)$ \\
\hline Mechanical thrombectomy & $57(1.2)$ & $841(0.9)$ \\
\hline Cardioversion & $61(1.3)$ & $59(0.1)$ \\
\hline Atrial fibrillation/flutter & $1.355(29.0)$ & $35479(37.6)$ \\
\hline Coronary heart disease & $2416(51.7)$ & $49272(52.2)$ \\
\hline Hypertension & $4432(94.8)$ & $89986(95.3)$ \\
\hline Diabetes mellitus & $2422(51.8)$ & $48560(51.4)$ \\
\hline Congestive heart failure & $1195(25.6)$ & $30551(32.4)$ \\
\hline Peripheral vascular disease & $1588(34.0)$ & $30459(32.3)$ \\
\hline Chronic kidney disease & $1200(25.7)$ & $27336(29.0)$ \\
\hline $\begin{array}{l}\text { Chronic obstructive } \\
\text { pulmonary disease }\end{array}$ & $1360(29.1)$ & $31797(33.7)$ \\
\hline Valvular heart disease & $1869(40.0)$ & $29958(31.7)$ \\
\hline Infective endocarditis & $82(1.8)$ & $175(0.2)$ \\
\hline Tobacco use & $974(20.8)$ & $17454(18.5)$ \\
\hline Alcohol abuse & $720(15.4)$ & $12954(13.7)$ \\
\hline
\end{tabular}

${ }^{*}$ Data are presented as number (\%) unless otherwise specified. †Data available for 5687 patients.

NIHSS, National Institutes of Health Stroke Scale; TEE, transesophageal echocardiography.

ventilation (HR, 1.0; 95\% CI 0.8 to 1.4). After adjustment for age, sex, race, and diagnosis of stroke versus TIA, TEE remained not associated with an increased risk of respiratory failure (HR, $0.8 ; 95 \% \mathrm{CI} 0.6$ to 1.1 ). This lack of association persisted after additional adjustment for Charlson comorbidities, intravenous thrombolysis, and mechanical thrombectomy (HR, $0.9 ; 95 \%$ CI 0.6 to 1.2 ) (table 3). In the fully adjusted model, we found no significant evidence of a difference in the association among patients who had ischemic stroke (HR, $0.9 ; 95 \%$ CI 0.6 to 1.2) versus TIA (HR, 0.8 ; $95 \%$ CI 0.1 to 5.4 ) (p value for interaction, 0.94).

In a sensitivity analysis excluding patients with infective endocarditis and patients undergoing cardioversion, there was no association between TEE and risk of respiratory failure (HR, $0.9 ; 95 \%$ CI 0.6 to 1.2 ). We also 
Table 2 Characteristics of Medicare beneficiaries who had ischemic stroke or transient ischemic attack, stratified by respiratory failure ( $5 \%$ national sample)

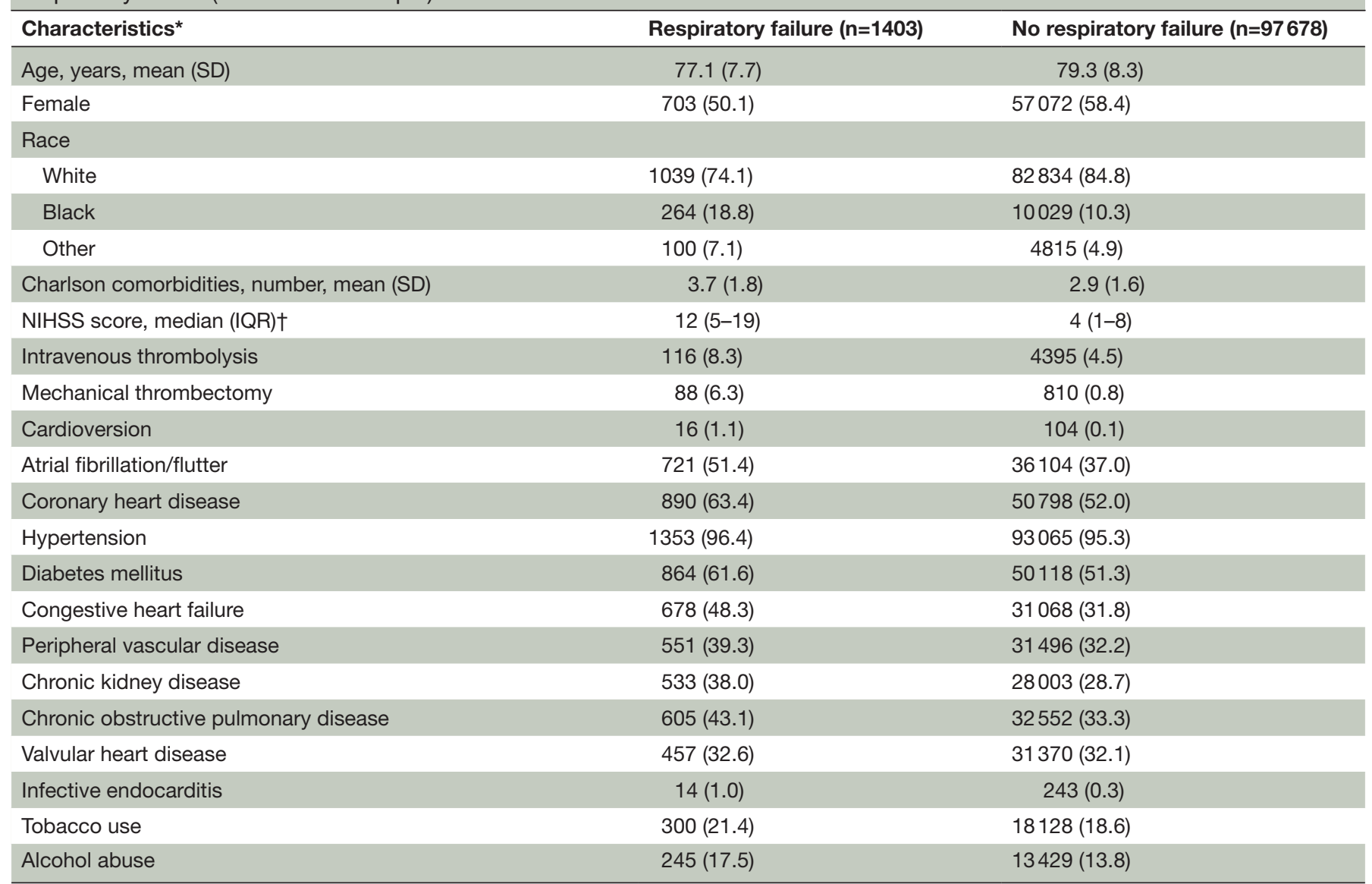

${ }^{*}$ Data are presented as number (\%) unless otherwise specified.

†Data available for 5687 patients.

NIHSS, National Institutes of Health Stroke Scale.

found no association between TEE and respiratory failure resulting in tracheostomy ( $\mathrm{HR}, 0.9 ; 95 \% \mathrm{CI} 0.5$ to 1.7 ). After excluding patients who died in the hospital or were discharged to hospice, there was similarly no association

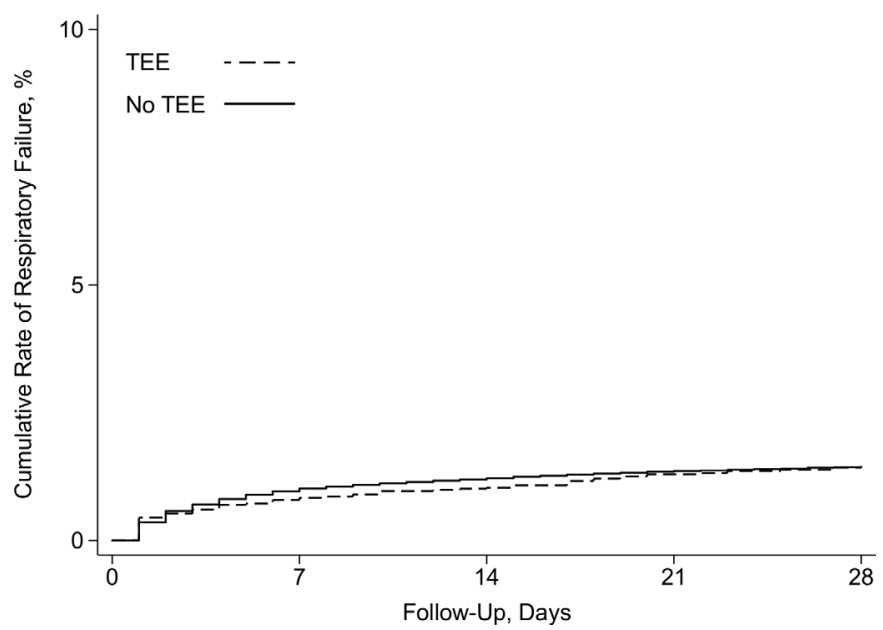

Figure 1 Cumulative risk of respiratory failure after ischemic stroke or transient ischemic stroke, stratified by transesophageal echocardiography (TEE). between TEE and respiratory failure (HR, 0.9; 95\% CI 0.6 to 1.4). In a model including an interaction term between TEE and calendar year, we found no significant evidence that the association between TEE and respiratory failure differed by year ( $p$ value for interaction, 0.75). Finally, when patients who experienced respiratory failure on the same day as TEE were included, the association between TEE and respiratory failure was not statistically significant, although the effect size was positive and of greater magnitude than in the primary analysis excluding those patients (HR, 1.3, 95\% CI 1.0 to 1.6).

\section{DISCUSSION}

In this IDEAL phase 4 study using a large sample of Medicare beneficiaries who had ischemic stroke and TIA, we found that TEE was not associated with an increased risk of respiratory failure after adjustment for demographics, comorbidities, stroke versus TIA diagnosis, and acute stroke treatments. In stratified analyses, this finding was similar in patients with stroke versus TIA. Our results were unchanged across multiple sensitivity analyses, including exclusion of patients with infective endocarditis and 
Table 3 Associations between TEE and respiratory failure among Medicare beneficiaries who had ischemic stroke or transient ischemic attack ( $5 \%$ national sample)

$\mathrm{HR}^{\star}(95 \% \mathrm{Cl})$

\begin{tabular}{ll}
\hline Primary models & \\
\hline Unadjusted & $1.0(0.8$ to 1.4$)$ \\
$\begin{array}{l}\text { Adjusted for age, sex, race, and } \\
\text { diagnosis of stroke versus transient } \\
\text { ischemic attack }\end{array}$ & $0.8(0.6$ to 1.1$)$ \\
$\begin{array}{l}\text { Also adjusted for comorbidities, } \\
\text { thrombolysis, and thrombectomy }\end{array}$ & $0.9(0.6$ to 1.2$)$ \\
$\begin{array}{l}\text { Subgroup analyses } \\
\begin{array}{l}\text { Patients who had ischemic stroke } \\
\text { Patients who had transient ischemic } \\
\text { attack }\end{array}\end{array}$ & $0.9(0.6$ to 1.2$)$ \\
\hline
\end{tabular}

Sensitivity analyses*

Excluding patients with endocarditis or 0.9 (0.6 to 1.2$)$ undergoing cardioversion

Outcome limited to respiratory failure $\quad 0.9$ (0.5 to 1.7) requiring tracheostomy

Excluding patients with in-hospital death or discharge to hospice

$0.9(0.6$ to 1.4$)$

Including TEE performed on the same

day as intubation

*All sensitivity analyses were based on model 3 from the primary models.

TEE, transesophageal echocardiography.

patients undergoing cardioversion, exclusion of patients who died during hospitalization or were discharged to hospice, limiting the outcome to respiratory failure requiring tracheostomy, including a TEE-calendar year interaction term to determine whether the relationship between TEE and respiratory failure changed over time, and including patients who experienced respiratory failure on the same day as TEE. Notably, the total incidence of respiratory failure was lower than the incidence reported in prior studies. ${ }^{17}$ This is most likely because patients requiring intubation on the day of admission were not included in our analysis as we were focused on respiratory failure occurring at least 1 day after TEE.

Data are scarce on the safety of TEE in regard to respiratory failure in the context of stroke or TIA. Several factors may increase risk of aspiration after TEE for patients who had stroke and TIA, including older age and frailty, dysphagia, ${ }^{18-21}$ use of sedating medications, ${ }^{22}$ and direct injury from probe manipulation during the procedure. ${ }^{23}$ Although prior studies have suggested TEE is generally safe and well tolerated in general cardiac populations, ${ }^{24-30}$ safety data in stroke and TIA have only been sparsely reported in relatively small, single-center studies. ${ }^{5}$ Recent evidence from studies not specifically enrolling patients who had stroke or TIA suggests that the risk of complications associated with TEE may be higher than previously suggested..$^{23}$ In this context, our results provide novel findings suggesting that the use of TEE for routine, community-onset stroke or TIA may not be associated with an increased risk of respiratory complications.

Our study has several limitations that should be considered when interpreting our results. First, our results were limited to patients with an admission diagnosis of stroke or TIA, and because we lacked data on the exact timing of strokes occurring during the hospitalization we could not examine the association between TEE and respiratory failure in patients who experienced in-hospital stroke or TIA. Second, our variables were defined using diagnosis and procedure codes, which carry a risk of misclassification. Third, we lacked data regarding the type, dosage, and depth of sedation in patients who underwent TEE. Fourth, the etiology of respiratory failure is often multifactorial and thus our results may be prone to residual confounding and selection bias. Fifth, all patients included in our study were at least 65 years of age and the results may not be generalizable to all age groups. Sixth, we do not have data on do-not-intubate orders, other requests for limitation of care, or the overall goals of care. It is possible that our definition of respiratory failure missed cases of respiratory failure which were not treated with invasive means because of more conservative goals of care, and such patients may also have been less likely to undergo other invasive procedures such as TEE. On the other hand, such patients with respiratory failure would presumably not have survived or been discharged to hospice, and our findings were unchanged in a sensitivity analysis excluding patients who died or were discharged to hospice. Seventh, for patients who experienced respiratory failure on the same day as TEE, we were unable to specify which event occurred first. Conservatively, we did not count TEE performed on the same day as respiratory failure to avoid results driven by cases in which TEE may have been performed in patients who were already intubated; however, this approach may also carry a risk of selection bias by excluding cases in which respiratory failure occurred during or immediately after TEE. Eighth, we did not have data on TEE operator technique or experience or how these variables may have changed over time. These unmeasured variables could confound the relationship between TEE and respiratory failure, although we found no evidence that this relationship changed by calendar year. Finally, a complete accounting of the risks and benefits associated with TEE was not possible with the data available as we do not know the full clinical indications for TEE in each case or the yield of the TEE studies.

In conclusion, we found that TEE was not associated with an increased risk of acute respiratory failure among older patients who had acute ischemic stroke or TIA. These findings may help clinicians weigh the potential risks and benefits of TEE for evaluation of ischemic stroke.

Twitter Art Sedrakyan @Artsytwits 
Contributors Conception and study design: SSB and HK. Data acquisition and analysis: $\mathrm{HK}$ and CZ. Drafting of the manuscript: SSB and HK. Manuscript revision for important intellectual content: BBN, JK, RBD, EJS, AS, and ID. Final approval of the manuscript: all authors. Study guarantor: HK.

Funding The authors have not declared a specific grant for this research from any funding agency in the public, commercial or not-for-profit sectors.

Competing interests HK serves as a PI for the NIH-funded ARCADIA trial (NINDS U01NS095869), which receives in-kind study drug from the BMS-Pfizer Alliance for Eliquis and ancillary study support from Roche Diagnostics, serves as Deputy Editor for JAMA Neurology, serves as a steering committee member of Medtronic's Stroke-AF trial (uncompensated), serves on an endpoint adjudication committee for a trial of empagliflozin for Boehringer Ingelheim, and has served on an advisory board for Roivant Sciences related to factor XI inhibition. BBN serves as a DSMB member for the PCORI-funded TRAVERSE trial and has received personal fees for medicolegal consulting on stroke.

Patient consent for publication Not required.

Ethics approval This study involves human participants. The Weill Cornell Medical College institutional review board approved this study (approval number 1507016424) and waived the requirement for informed consent

Provenance and peer review Not commissioned; externally peer reviewed.

Data availability statement Data may be obtained from a third party and are not publicly available. The data used for this analysis cannot be directly shared by the authors under the terms of their data use agreement, but the data can be obtained by application to CMS.

Open access This is an open access article distributed in accordance with the Creative Commons Attribution Non Commercial (CC BY-NC 4.0) license, which permits others to distribute, remix, adapt, build upon this work non-commercially, and license their derivative works on different terms, provided the original work is properly cited, appropriate credit is given, any changes made indicated, and the use is non-commercial. See: http://creativecommons.org/licenses/by-nc/4.0/.

ORCID iDs

Art Sedrakyan http://orcid.org/0000-0003-3882-9765

Hooman Kamel http://orcid.org/0000-0002-5745-0307

\section{REFERENCES}

1 Hernandez Fustes OJ, Arteaga Rodriguez C, Hernandez Fustes OJ. In-Hospital mortality from cerebrovascular disease. Cureus 2020;12:e8652

2 Barlas RS, Clark AB, Bettencourt-Silva JH, et al. Pneumonia and risk of serious adverse outcomes in hospitalized strokes in Thailand. $J$ Stroke Cerebrovasc Dis 2019;28:1448-54.

3 Giruparajah M, Bosch J, Vanassche T, et al. Global survey of the diagnostic evaluation and management of cryptogenic ischemic stroke. Int J Stroke 2015;10:1031-6.

4 Khalid O, Srivastava R, Mulhall A, et al. Conscious sedation: for a TEE, is it always required? Echocardiography 2010;27:74-6.

5 McGrath ER, Paikin JS, Motlagh B, et al. Transesophageal echocardiography in patients with cryptogenic ischemic stroke: a systematic review. Am Heart J 2014;168:706-12.

6 De Castro S, Rasura M, Di Angelantonio E, et al. Distribution of potential cardiac sources of embolism in young and older stroke patients: implications for recurrent vascular events. J Cardiovasc Med 2006;7:191-6.

7 Pop G, Sutherland GR, Koudstaal PJ, et al. Transesophageal echocardiography in the detection of intracardiac embolic sources in patients with transient ischemic attacks. Stroke 1990;21:560-5.

8 Fukujima MM, Tatani SB, Aguiar AS, et al. Transesophageal echocardiography discloses unexpected cardiac sources of embolus in stroke patients aged more than 45 years. Arq Neuropsiquiatr 2005;63:941-5

9 Censori B, Colombo F, Valsecchi MG, et al. Early transoesophageal echocardiography in cryptogenic and lacunar stroke and transient ischaemic attack. J Neurol Neurosurg Psychiatry 1998;64:624-7.

10 Center for Medicare and Medicaid Services. Medicare limited dataset files 2019.

11 Tirschwell DL, Longstreth WT. Validating administrative data in stroke research. Stroke 2002;33:2465-70.

12 Andrade SE, Harrold LR, Tjia J, et al. A systematic review of validated methods for identifying cerebrovascular accident or transient ischemic attack using administrative data. Pharmacoepidemiol Drug Saf 2012;21 Suppl 1:100-28.

13 Kokotailo RA, Hill MD. Coding of stroke and stroke risk factors using International classification of diseases, revisions 9 and 10. Stroke 2005;36:1776-81.

14 Goldman LE, Chu PW, Osmond D, et al. The accuracy of presenton-admission reporting in administrative data. Health Serv Res 2011;46:1946-62.

15 Quan H, Li B, Saunders LD, et al. Assessing validity of ICD-9-CM and ICD-10 administrative data in recording clinical conditions in a unique dually coded database. Health Serv Res 2008;43:1424-41.

16 Saber H, Saver JL. Distributional validity and prognostic power of the National Institutes of health stroke scale in US administrative claims data. JAMA Neurol 2020;77:606-12.

17 Lahiri S, Mayer SA, Fink ME, et al. Mechanical ventilation for acute stroke: a multi-state population-based study. Neurocrit Care 2015;23:28-32.

18 González-Fernández M, Ottenstein L, Atanelov L, et al. Dysphagia after stroke: an overview. Curr Phys Med Rehabil Rep 2013;1:187-96.

19 Martino R, Foley N, Bhogal S, et al. Dysphagia after stroke: incidence, diagnosis, and pulmonary complications. Stroke 2005;36:2756-63.

20 Smithard DG, O'Neill PA, England RE, et al. The natural history of dysphagia following a stroke. Dysphagia 1997;12:188-93.

21 Smithard DG, O'Neill PA, Park C, et al. Complications and outcome after acute stroke. Stroke 1996;27:1200-4

22 Green SM, Mason KP, Krauss BS. Pulmonary aspiration during procedural sedation: a comprehensive systematic review. $\mathrm{Br} J$ Anaesth 2017;118:344-54.

23 Freitas-Ferraz AB, Bernier M, Vaillancourt R, et al. Safety of transesophageal echocardiography to guide structural cardiac interventions. J Am Coll Cardiol 2020;75:3164-73.

24 Piercy M, McNicol L, Dinh DT, et al. Major complications related to the use of transesophageal echocardiography in cardiac surgery. $J$ Cardiothorac Vasc Anesth 2009;23:62-5.

25 Huang C-H, Lu C-W, Lin T-Y, et al. Complications of intraoperative transesophageal echocardiography in adult cardiac surgical patients - experience of two institutions in Taiwan. J Formos Med Assoc 2007;106:92-5.

26 Sainathan S, Andaz S. A systematic review of transesophageal echocardiography-induced esophageal perforation. Echocardiography 2013;30:977-83.

27 Hilberath JN, Oakes DA, Shernan SK, et al. Safety of transesophageal echocardiography. J Am Soc Echocardiogr 2010;23:1115-27.

28 Purza R, Ghosh S, Walker C, et al. Transesophageal echocardiography complications in adult cardiac surgery: a retrospective cohort study. Ann Thorac Surg 2017;103:795-802.

29 Kallmeyer IJ, Collard CD, Fox JA, et al. The safety of intraoperative transesophageal echocardiography: a case series of 7200 cardiac surgical patients. Anesth Analg 2001;92:1126-30.

30 Lennon MJ, Gibbs NM, Weightman WM, et al. Transesophageal echocardiography-related gastrointestinal complications in cardiac surgical patients. J Cardiothorac Vasc Anesth 2005;19:141-5.

31 Freitas-Ferraz AB, Rodés-Cabau J, Junquera Vega L, et al. Transesophageal echocardiography complications associated with interventional cardiology procedures. Am Heart J 2020;221:19-28. 